\title{
BMJ Open Does physiological distribution of blood parameters in children depend on socioeconomic status? Results of a German cross-sectional study
}

\author{
Kristin Rieger, ${ }^{1}$ Mandy Vogel, ${ }^{2}$ Christoph Engel, ${ }^{3}$ Uta Ceglarek, ${ }^{4}$ Kristian Harms, ${ }^{4}$ \\ Ulrike Wurst, ${ }^{1}$ Holger Lengfeld, ${ }^{5}$ Matthias Richter, ${ }^{6}$ Wieland Kiess ${ }^{1,2}$
}

To cite: Rieger K, Vogel M, Engel C, et al. Does physiological distribution of blood parameters in children depend on socioeconomic status? Results of a German cross-sectional study. BMJ Open 2018;8:e019143. doi:10.1136/ bmjopen-2017-019143

- Prepublication history and additional material for this paper are available online. To view these files, please visit the journal online (http://dx.doi. org/10.1136/bmjopen-2017019143).

Received 13 August 2017 Revised 5 December 2017 Accepted 30 January 2018

Check for updates

For numbered affiliations see end of article.

Correspondence to Ms. Kristin Rieger; kristin.rieger@medizin.unileipzig.de

\section{ABSTRACT}

Objectives In the present study, we examined the relation between socioeconomic status (SES) and the physiological distribution of iron-related blood parameters.

Design This is a cross-sectional analysis of longitudinal population-based cohort study.

Setting Based on a sample of healthy participants from a German research centre, various blood parameters and values of clinical examinations and questionnaires were collected.

Participants A total of 1206 healthy volunteers aged 2.5 to 19 years, one child per family randomly selected, were included.

\section{Primary and secondary outcome}

measures Associations between the SES of children by Winkler-Stolzenberg Index (WSI) and its dimensions (income, education, occupation) and iron-related blood parameters (haemoglobin, ferritin and transferrin) were analysed by linear regression analyses. Gender and pubertal stage were included as covariables. Additionally, associations between SES of children by WSI and physical activity (side-to-side jumps, push-ups) as well as body mass index (BMI) were analysed by linear regression analyses.

Results Children with high WSI or family income showed significantly increased z-scores for haemoglobin $(P=0.046 ; P<0.001)$. Children with increased WSI or family income showed significantly lower z-scores for transferrin $(\mathrm{P}<0.001)$. There was a significant correlation between haemoglobin and gender $(P<0.001)$ and between transferrin and pubertal stage $(\mathrm{P}=0.024)$. Furthermore, physical activity was positively correlated and BMI was negatively correlated with WSI $(\mathrm{P}<0.001)$.

Discussion Our data show an association between SES and the distribution of iron-dependent parameters. Lower SES is correlated with lower values for haemoglobin and higher values for transferrin. Furthermore, we demonstrate that physical activity and BMI are associated with SES. Whereas higher SES is correlated with higher values for physical activity and lower BMI. Our parameters are standardised as z-scores with the advantages that the results are comparable across different age groups and present physiological courses.

Trial registration number NCT02550236; Results.

\section{Strengths and limitations of this study}

- The aim was to detect changes in the physiological distribution of iron-related blood parameters, depending on the socioeconomic status (SES) before manifestation of anaemia to exploit a preventive potential.

- Laboratory parameters were standardised as z-scores, which allow a comparison across different age groups.

- A cohort of healthy children from different social strata was available.

- The study population is unequally distributed with over-representation of families with medium SES.

- Other laboratory parameters important for clinical practice, such as transferrin saturation, were not collected in the present cohort.

\section{INTRODUCTION}

Previous studies from Germany and other countries show that individuals with lower education, occupational position and income have a decreased life expectancy and suffer more often from acute and chronic diseases compared with individuals with a more favourable socioeconomic status (SES). ${ }^{1}$ However, until today research on socioeconomic inequalities in health has largely focused on adults and the elderly. Only recently has a growing interest and global focus on child and adolescent health emerged. ${ }^{2}{ }^{3}$ The existing evidence shows a marked social gradient for several child health outcomes. For example, children of families with a low SES have a higher risk of premature mortality, low birth weight, congenital anomalies, nutritive deficits, infections, psychomotor disorders, limitations in cognitive development and severe injuries. ${ }^{4-7}$

According to WHO, a major health problem in childhood is micronutrient deficiency, especially of iron, often resulting in 
anaemia. ${ }^{68}$ Anaemia is defined as low-blood haemoglobin and decreased red blood cells. The global prevalence of anaemia amounts to $39 \%$ in preschool children and $46 \%$ in children aged 5 to $14 .{ }^{9}$ Half of the cases are caused by nutritional iron deficiency. ${ }^{9}$ Unlike adults, iron deficiency in children manifests early due to the increased demand in adolescence. In adolescence, girls are especially threatened in case of menstrual blood loss. ${ }^{10}$ Major symptoms are immune deficiency, growth retardation, fatigue, changes in hormone balance as well as cognitive, physical and behavioural deficits. ${ }^{12} 1112$ Regarding the manifestation, genetic factors have less influence than external biological factors, for example, exposure to toxins or pollutants. ${ }^{3}$ Various studies have highlighted the role of different social factors in the development of anaemia, such as school education, income, housing conditions, sanitary facilities, number of siblings, obesity, physical activity and quality of medical care. ${ }^{513-15}$ Nevertheless, two questions remain to be answered: Are there objective parameters in iron metabolism other than haemoglobin that might be associated with socioeconomic characteristics? Are there relevant differences in blood iron parameters depending on socioeconomic characteristics before the manifestation of anaemia? So far, only one study from Germany has examined the association between objective laboratory parameters of health, for example, haemoglobin and ferritin and parental income. ${ }^{16}$ Here, no significant correlation could be shown for haemoglobin, whereas a significant correlation was visible between low ferritin levels and low parental income. ${ }^{16}$

Therefore, the aim of our study was to analyse differences in laboratory measures of iron metabolism due to socioeconomic effects before the manifestation of anaemia. We examined haemoglobin, ferritin and transferrin as laboratory parameters, as those are commonly used in diagnostics of iron deficiency. ${ }^{17}$ Additionally, we examined if SES is associated with physical activity or body mass index (BMI).

\section{METHODS}

\section{Study population}

Our study was based on a sample of participants from the Leipzig Research Centre for Civilization Diseases (LIFE Child), consisting of healthy volunteers aged 2.5 to 19 years. LIFE Child is a large prospective, longitudinal, population-based cohort study of urban children from fetal life until adulthood (see online supplementary files 1 and 2). The study collects detailed information from clinical examinations, questionnaires and interviews as well as a collection of several types of biological materials at various time points (see online supplementary file 3 ). Participants have been followed annually over a period of 10 years, irrespective of the age at the first participation in the study. Children were recruited from a community-based, collaborative network of university hospitals, local clinics, public health centres, kindergartens, schools and partner study centres like the Integrated Research and Treatment Centre for Obesity with the inclusion criteria of healthiness. ${ }^{18}$

In our study population, children and adolescents were recruited from 2011 until 2015. Various blood and socioeconomic parameters were collected at different time points. Initially the cohort consisted of families, some of whom participated with several children. To ensure the independence of the measuring points, only one child per family was randomly selected by using random number generator as implemented in the $\mathrm{R}$ base package. The statistical software $\mathrm{R}$ is introduced below. At the first time point, we analysed values of haemoglobin, ferritin, transferrin and SES measures cross-sectionally. In total, 1206 probands could be included in case of complete data sets for haemoglobin, ferritin, transferrin and Winkler-Stolzenberg Index (WSI). None of the children had iron substitution or a haematological disease. All tests done on human subjects described herein have been carried out with the consent of the competent ethics committee, in accordance with national law, and the 1975 Declaration of Helsinki (in the current, amended version). All parents of involved test subjects have signed a consent form.

\section{Laboratory measures}

Venous blood samples were obtained in EDTA whole blood and Serum-Monovettes (Sarstedt, Nuembrecht, Germany). Fasting blood samples were not a necessary according to the requirements of the Committee of Scientific Medical Expert Associations (AWMF) ${ }^{19}$ or WHO. ${ }^{20}$ There were no icteric, lipemic or haemolytic samples. Haemoglobin was determined with the sodium lauryl sulfate haemoglobin method (SLS detection method). Transferrin was determined by immunological turbidity tests (c module), and ferritin was determined with electrochemiluminescence immunoassays (e module) (each Roche Diagnostics , Mannheim, Germany) with the Cobas 8000.

The determination of the number of erythrocytesnecessary for calculating the mean corpuscular volume (MCV) and mean corpuscular haemoglobin (MCH) was carried out with impedance measurement and hypodynamic focusing, at the same time the haematocrit was calculated by cumulative pulse height summation (each Sysmex XN-9000, Sysmex Germany, Norderstedt, Germany). MCV can be calculated by the division of haematocrit through the number of erythrocytes and $\mathrm{MCH}$ by the division of haemoglobin concentration in the blood through the number of erythrocytes. C reactive protein was measured by latex-enhanced immunoturbidimetric test with Cobas analyser series (photometric measuring unit, c-module, Roche Diagnostics).

Based on the AWMF guideline for iron deficiency diagnostics in children, ${ }^{19}$ states of iron deficiency were categorised as follows: latent iron deficiency (decrease in ferritin with normal haemoglobin, MCV and $\mathrm{MCH}$ ), manifest iron deficiency (decrease in haemoglobin and $\mathrm{MCV}$ ). Anaemia of other cause was defined as age-related 
median haemoglobin decreased by more than $2 \mathrm{SDs},{ }^{19}$ excluding manifest iron deficiency as mentioned above.

\section{Indicators of SES, BMI, puberty and physical activity}

SES was measured by the WSI, a commonly used instrument in German socioepidemiological research. ${ }^{21}{ }^{22}$ The WSI was designed as a proxy to measure the SES of a family. The WSI includes items on parental school education/occupational qualification (ie, from 1 point equalling no school education or professional qualification to 7 points equalling university level education), occupational status (ie, from 1 point equalling unemployed to 7 points equalling executive status) of both parents and net household income per month (ie, from 1 point meaning lower than $€ 1250$ to 7 points meaning higher than $€ 5000$ ). Out of these, the multidimensional WSI was calculated as a sum score of the above-mentioned indicators. If WSI values of both parents were obtained, the higher parental score was taken as the final value. SES was scaled into the following categories according to the total points of the WSI: low SES at 3-8 points, medium SES at 9-14 points and high SES at 15-21 points. ${ }^{23}$ These categories were used for descriptive analysis. For regression analyses, total WSI score and the individual WSI dimensions were used on a metric scale.

Height was measured with the stadiometer ('Prof. Keller', Längenmesstechnik Limbach, Limbach-Oberfrohna, Germany) with a measurement accuracy of $0.10 \mathrm{~cm}$. The participants were weighed with the 'seca 701' scale (seca, Hamburg, Germany) which is accurate to $50 \mathrm{~g}$. BMI can be calculated by the division of weight in kilograms through size in metre squared.

The pubertal stage was assessed according to Tanner stages $^{24} 25$ by specially trained and regularly instructed investigators. However, there is only information of 920 of the participating 1206 children collected. The Tanner stages were separated into three groups (Tanner stage 1 , stages 2-4 and stage 5, reflecting prepubertal, pubertal and postpubertal stages) to emphasise on puberty-specific changes.

Physical activity was measured as physical fitness test as part of the motoric module (MoMo). MoMo is a submodule of the German Health Interview and Examination Survey for Children and Adolescents conducted by the Robert Koch Institute. This consists of exercises in the areas of endurance, strength, agility, coordination and flexibility. ${ }^{26}$ We especially picked coordination and strength as parameters for our study. The jumping side-to-side test was used to assess gross motor coordination under time constraint. The subjects were asked to perform as many jumps from side to side as possible during two $15 \mathrm{~s}$ intervals, and the numbers for the two intervals were averaged. Dynamic strength endurance of the upper extremities was assessed using the push-up test. The subjects were asked to do as many push-ups as possible within $40 \mathrm{~s}^{27}$ However, there is only information of 752 participants for side-to-side jumps and of 715 participants for push-ups.

\section{Statistics}

Data analysis was performed with the free statistical software R V.3.1.2 (see online supplementary file 4). Values for haemoglobin, ferritin, transferrin, BMI and physical activity were standardised as age-adapted z-scores (SD score). The z-score system expresses the value as a number of SDs below or above the reference mean or median value. Z-scores are directly related to percentiles (z-score 0 corresponds to P50, z-score -1.881 corresponds to P3, z-score 1.881 corresponds to P97). Z-scores were calculated by a standardised formula ${ }^{28}: \mathrm{z}$-score $=($ observed value-median value of the reference population)/ $\mathrm{SD}$ deviation value of reference population. These z-scores were calculated by a modified lamda,mu,sigma (LMS)) method according to Cole et al taken from the WHO recommendations, ${ }^{29}{ }^{30}$ which is also implemented in the GAMLSS package of the statistics $\mathrm{R}$ software. ${ }^{31-33}$ The approach of estimating the parameters as a continuous function of age was preferred, because it provided a better model for physiological development than a stepby-step consideration of different age intervals ${ }^{29}$ and is also recommended by WHO. ${ }^{34}$ Detailed information of this complex resampling technique has been described in previous studies. ${ }^{356}$ Raw WSI and its dimensions considered as metric scales.

First, descriptive analyses of the study population were performed concerning age groups, SES categories, pubertal stage and mean z-scores for haemoglobin, ferritin, transferrin and BMI according to age. Significance of associations between the laboratory parameters and gender, pubertal stage and BMI was proved. Furthermore, we analysed the absolute and relative proportion of latent iron deficiency, manifest iron deficiency and anaemia of other cause. We proved its significance in association to WSI by logistic regression analyses. Second, regression analyses were carried out for each target variable: haemoglobin, ferritin and transferrin. Different models were created with WSI and its dimensions (parental school education/occupational qualification, occupational status and net household income) as predictors. In bivariate regression analyses, each target variable and total WSI were included (model 1). Furthermore, multivariate regression analyses including all dimensions of the WSI, pubertal stage and gender were carried out (model 2). Where pubertal stage does not deliver any information beyond the information of the other variables, it was removed. Additionally, we tested a model for WSI as target variable and pubertal stage, BMI and physical activity (side-to-side jumps, push-ups) as predictors (model 3). All models were tested for interactions between the variables. When there was no interaction, the interaction term was removed from the model.

\section{RESULTS}

\section{Characteristics of the study population}

Characteristics of the study population are presented in table 1 . The largest age group was from 6 to under 


\begin{tabular}{|c|c|c|c|}
\hline Characteristics & $\begin{array}{l}\text { Boys } \\
(n=635)\end{array}$ & Girls $(n=571)$ & $\begin{array}{l}\text { Total } \\
(n=1206)\end{array}$ \\
\hline \multicolumn{4}{|c|}{ Age categories in years, $\mathrm{n}(\%)$} \\
\hline $2.5-<6$ & 95 & 82 & $177(14.7)$ \\
\hline $6-<12$ & 296 & 242 & $538(44.6)$ \\
\hline $12-19$ & 244 & 247 & $491(40.7)$ \\
\hline \multicolumn{4}{|c|}{ Pubertal stage, n (\%) } \\
\hline Prepubertal & 260 & 219 & $479(39.7)$ \\
\hline Pubertal & 121 & 200 & $321(26.6)$ \\
\hline Postpubertal & 19 & 101 & $120(10.0)$ \\
\hline Missing & 235 & 51 & $286(23.7)$ \\
\hline \multicolumn{4}{|c|}{ Blood parameter z-scores, mean value (SD) } \\
\hline Haemoglobin*† & $0.17(1.01)$ & $-0.20(0.95)$ & $-0.006(1.0)$ \\
\hline Ferritin* & $0.06(0.98)$ & $-0.07(1.02)$ & $0.0001(1.0)$ \\
\hline Transferrin† & $-0.01(1.0)$ & $0.03(1.0)$ & $0.008(1.0)$ \\
\hline $\begin{array}{l}\text { BMI z-score, mean } \\
\text { value (SD) }\end{array}$ & $0.21(1.16)$ & $0.29(1.23)$ & $0.25(1.2)$ \\
\hline \multicolumn{4}{|c|}{ SES of parents, $n(\%) \ddagger$} \\
\hline Low & 82 & 72 & $154(12.8)$ \\
\hline Medium & 280 & 252 & $532(44.1)$ \\
\hline High & 273 & 247 & $520(43.1)$ \\
\hline
\end{tabular}

*Significant differences for gender.

†Significant differences for pubertal stage, $\mathrm{P}<0.05$

(haemoglobin was negatively correlated, transferrin was positively correlated).

‡Categories of SES result from individual Winkler-

Stolzenberg Index sums: 3 to 8 scores-low stratum, 9 to 14 scores-middle stratum, 15 to 21 scores-high stratum.

BMI, body mass index; SES, socioeconomic status.

12 years of age $(44.6 \%)$, followed by adolescence (12 to 19 years) $(40.7 \%)$ and preschoolers aged 2.5 to under 6 years $(14.7 \%)$. Children of parents with medium SES were the largest group (44.1\%), followed by children of parents with high SES (43.1\%) and low SES (12.8\%).
Pubertal stage was reached in $76.3 \%$ of the participants. Children with prepubertal stage were the strongest group $(39.1 \%)$, followed by pubertal stage $(26.6 \%)$ and postpubertal stage $(10.0 \%)$. Slightly more boys than girls were included (boys $52.7 \%$, girls $47.3 \%$ ). Haemoglobin $(\mathrm{P}<0.001$; mean $\mathrm{z}$-score: boys 0.17 , girls -0.20$)$ and ferritin ( $\mathrm{P}=0.018$; mean z-score: boys 0.06 , girls -0.07 ) were significantly lower for girls than for boys, whereas transferrin z-scores were not significantly higher in boys (boys -0.01 , girls 0.03 ). Mean BMI-z-score was not significantly lower for boys than for girls (boys 0.21 , girls 0.29 ) (table 1). The distribution of the laboratory parameters in each SES category is shown in figure 1. The mean values of haemoglobin z-scores significantly increased by 0.225 from low to high SES $(\mathrm{P}=0.014)$. The mean ferritin z-scores were $-0.15,0.004$ and 0.001 , respectively, for low, medium and high SES. This distribution had no significant effect $(\mathrm{P}=0.863)$. Furthermore, mean values of transferrin z-scores decreased significantly with rising SES from 0.246 to $-0.075(\mathrm{P}<0.001)$. Anaemia in general was shown for $4.2 \%$ of the children, whereas $0.7 \%$ showed a manifest iron deficiency and $3.6 \%$ had anaemia of other cause. Furthermore, $6.9 \%$ showed a latent iron deficiency. In logistic regression analyses, anaemia of other cause $(\mathrm{P}=0.032)$ and anaemia, in total, was proven as significant association to lower WSI $(\mathrm{P}=0.013)$.

\section{Regression models}

Model 1: Bivariate regression analyses of model 1 are presented in table 2. Haemoglobin was significantly and positively associated with rising WSI $(\mathrm{P}=0.046)$. Ferritin was not significantly related to WSI. Decreasing transferrin was significantly associated with increasing WSI $(\mathrm{P}<0.001)$.

Model 2: Multivariate regression analyses of model 2 are presented in table 3 . Haemoglobin is significantly positively correlated with income $(\mathrm{P}<0.001)$. Additionally, haemoglobin is significantly higher in boys than in girls $(\mathrm{P}<0.001)$. Ferritin was significantly positively correlated with income $(\mathrm{P}=0.009)$. Furthermore,
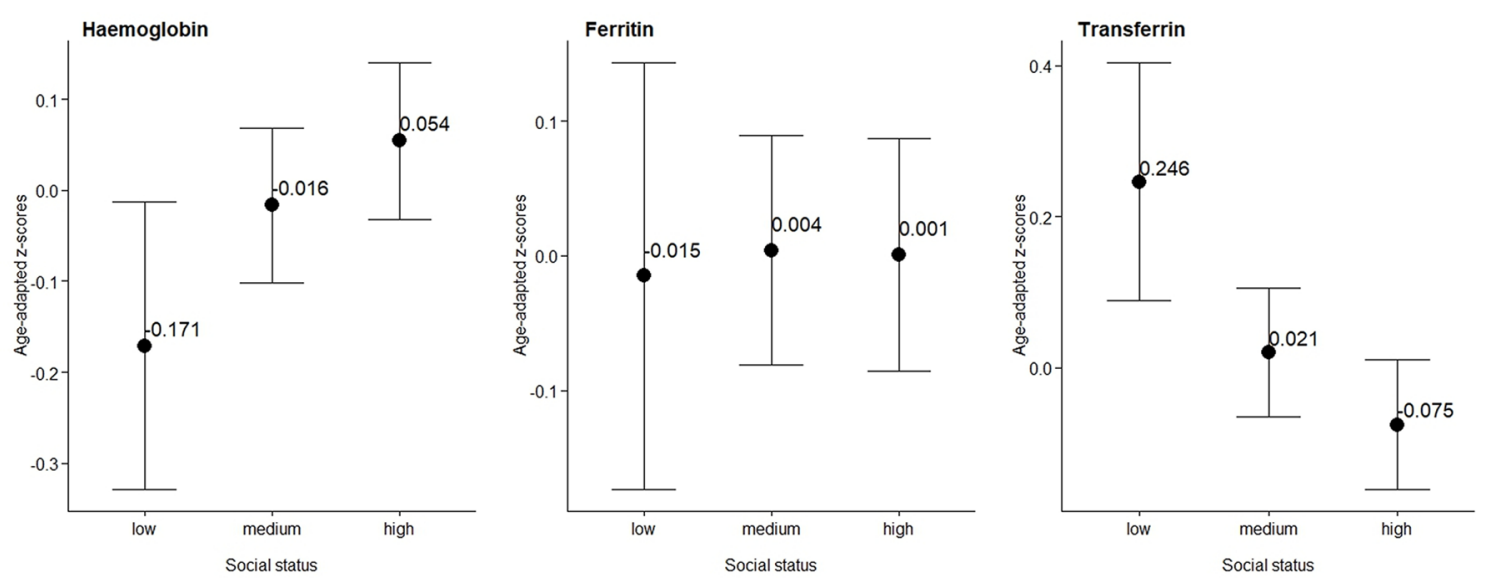

Figure 1 Comparison of the levels of the socioeconomic status (low, medium, high) according to age-adapted z-scores of haemoglobin, ferritin and transferrin. The points depict the means of each group, precisely labelled. Statistical significance was reached for haemoglobin $(\mathrm{P}=0.014)$ and transferrin $(\mathrm{P}<0.001)$. 
Table 2 Summary of the results of regression model 1 (bivariate regression analyses for each target value (haemoglobin, ferritin, transferrin) with WSI)

\begin{tabular}{|c|c|c|c|}
\hline Regression models & $B^{\star}$ & $\mathrm{Cl}$ & $P \ddagger$ \\
\hline \multicolumn{4}{|l|}{ Haemoglobin } \\
\hline WSI & 0.013 & 0.0002 to 0.0260 & 0.046 \\
\hline \multicolumn{4}{|l|}{ Ferritin } \\
\hline WSI & 0.002 & -0.0111 to 0.0149 & 0.774 \\
\hline \multicolumn{4}{|l|}{ Transferrin } \\
\hline WSI & -0.026 & -0.0389 to -0.0131 & $<0.001$ \\
\hline
\end{tabular}

Haemoglobin, ferritin and transferrin as target variables. WSI as predictor. Significant correlations are bold.

${ }^{*} \mathrm{~B}$ indicates the regression coefficient,

$+95 \% \mathrm{Cl}$ for $\mathrm{B}$

$\ddagger$ Bilateral significance $\mathrm{P}<0.05$.

WSI, Winkler-Stolzenberg Index.

transferrin was significantly negatively correlated with income $(\mathrm{P}=0.008)$ and positively correlated with pubertal stage $(\mathrm{P}=0.024)$.

\begin{tabular}{|c|c|c|c|}
\hline $\begin{array}{l}\text { Regression } \\
\text { models }\end{array}$ & $\mathbf{B}^{*}$ & $\mathrm{Cl}+$ & $P \ddagger$ \\
\hline \multicolumn{4}{|l|}{ Haemoglobin§ } \\
\hline Income & 0.086 & 0.0455 to 0.1259 & $<0.001$ \\
\hline Education & -0.033 & -0.0752 to 0.0096 & 0.130 \\
\hline Occupation & 0.001 & -0.0418 to 0.0443 & 0.955 \\
\hline Gender & -0.349 & -0.4712 to -0.2277 & $<0.001$ \\
\hline \multicolumn{4}{|l|}{ Ferritin§ } \\
\hline Income & 0.054 & 0.0137 to 0.0951 & 0.009 \\
\hline Education & -0.036 & -0.0794 to 0.0064 & 0.096 \\
\hline Occupation & -0.001 & -0.0442 to 0.0429 & 0.977 \\
\hline Gender & -0.111 & -0.2341 to 0.0123 & 0.078 \\
\hline \multicolumn{4}{|l|}{ Transferrin } \\
\hline Income & -0.062 & -0.1088 to -0.0162 & 0.008 \\
\hline Education & -0.025 & -0.0751 to 0.0249 & 0.325 \\
\hline Occupation & -0.004 & -0.0541 to 0.0467 & 0.885 \\
\hline Pubertal stage & 0.261 & 0.0351 to 0.4873 & 0.024 \\
\hline Gender & -0.022 & -0.1679 to 0.1238 & 0.766 \\
\hline
\end{tabular}

Haemoglobin, ferritin and transferrin as target variables. Net household income, highest education, occupational status, pubertal stage and gender as predictors. Significant correlations are bold.

${ }^{*} \mathrm{~B}$ indicates the regression coefficient.

† $95 \% \mathrm{Cl}$ for $\mathrm{B}$.

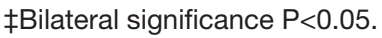

§Pubertal stage gives no information beyond the information of the other variables; therefore, it was removed from the analyses.

WSI, Winkler-Stolzenberg Index.
Table 4 Summary of the results of regression model 3 (bivariate regression analyses for each target value (side-toside jumps, push-ups, BMI) with WSI)

\begin{tabular}{|c|c|c|c|}
\hline Regression models & $\mathrm{B}^{*}$ & Cl† & $\mathbf{P} \ddagger$ \\
\hline \multicolumn{4}{|l|}{ Side-to-side jumps } \\
\hline WSI & 0.057 & 0.0384 to 0.0749 & $<0.001$ \\
\hline \multicolumn{4}{|l|}{ Push-ups } \\
\hline WSI & 0.050 & 0.0332 to 0.0672 & $<0.001$ \\
\hline \multicolumn{4}{|l|}{ BMI } \\
\hline WSI & -0.055 & -0.070 to -0.0399 & $<0.001$ \\
\hline
\end{tabular}

Side-to-side jumps, push-ups and BMI as target variables. WSI as predictor. Significant correlations are bold.

${ }^{*} \mathrm{~B}$ indicates the regression coefficient.

$+95 \% \mathrm{Cl}$ for $\mathrm{B}$.

$\ddagger$ Bilateral significance $\mathrm{P}<0.05$.

BMI, body mass index; WSI, Winkler-Stolzenberg Index.

Model 3: Bivariate regression analyses of model 3 are presented in table 4 . Side-to-side jumps are positively correlated with WSI $(\mathrm{P}<0.001)$. Push-ups are positively correlated with WSI $(\mathrm{P}<0.001)$. BMI is negatively correlated with WSI $(\mathrm{P}<0.001)$.

\section{DISCUSSION}

Our study is the first to show that the distribution of iron-related laboratory parameters is associated with parental SES in children and adolescents. We demonstrate that haemoglobin is positively correlated with WSI, income and gender, and ferritin is positively correlated with income. In contrast, we demonstrate negative correlations of transferrin with WSI and income and positive correlation with pubertal stage. Furthermore, we evaluated that rising WSI is associated with higher values of dynamic strength endurance (push-ups), gross motor coordination (side-to-side jumps) and with lower BMI. A special feature of our study is that the laboratory parameters, the parameters for physical activity and BMI are standardised as z-scores. Interpreting the results in terms of z-scores is in accordance to the recommendations of $\mathrm{WHO}^{28} 34$ and has several advantages: z-scores have the same statistical relation to the distribution of the reference around the mean at all ages, which makes results comparable across age groups, are directly related to percentiles and yield a better representation of physiological processes. ${ }^{35} 36$

Children with lower SES have lower concentrations of haemoglobin and higher concentrations of transferrin compared with individuals with higher SES (figure 1). These findings support the fact that differences in health outcomes depending on SES, which is in accordance with existing data on other parameters of health. ${ }^{163738} \mathrm{~A}$ combination of low haemoglobin and ferritin and high transferrin are typically for iron deficiency. However, there are defined age and gender-specific limits to determine iron deficiency, ${ }^{17} 20$ which were in our study 
fulfilled by a minority. Nevertheless, we proved significant correlation between decreased WSI and the occurrence of anaemia caused by manifest iron deficiency and other causes. Therefore, we can state that WSI is associated with differences of the distribution of iron-related parameters and the appearance of anaemia.

In our study, income presents the strongest predictor for values of haemoglobin and transferrin. One possible explanation for this association might be related to nutrition. Children of families of very low SES may be undernourished because they do not receive enough food. Accessing healthy foods at a feasible price is a challenge and may compel families to make food choices that are contrary to current nutritional guidance. ${ }^{39} 40$ Previous studies show that restricted food budgets are insufficient for ensuring healthy diets. ${ }^{39} 40$ Thus, diet quality is directly influenced by income. Other cross-sectional and longitudinal analyses support the hypothesis of the influence of income on alimentation via budget/prices for healthy food. ${ }^{41-43}$ However, monetary shortages are not the only cause of an unhealthy diet. Low-income individuals also have a higher burden of employment-related, food-related, and housing-related insecurity. This leads to increased levels of stress, which often results in eating as a coping mechanism, as well as an increased propensity to choose unhealthy foods. ${ }^{424-46}$ Moreover, it can be emphasised that stress reduces gastrointestinal absorption and the synthesis of haemoglobin, ferritin and transferrin in the spleen, bone marrow or liver by inducing inflammation. ${ }^{47}$ In LIFE Child, a food frequency questionnaire is implemented, we had to consider whether to include it in our study. We decided against it, while participation was low and validity showed fair to moderate ranking except for pasta/rice and white bread and the ability to assess absolute intakes is limited. ${ }^{48}$ However, we had the possibility to include a motoric module. As a result, we could show that higher values for dynamic strength endurance and gross motor coordination are associated with rising WSI. Income influences physical activity, since many activities, that is, in sports clubs, are fee based and therefore not affordable for families with low income. A German study demonstrates that a low family income and high degree of physical inactivity are positively associated with obesity. ${ }^{14}$ In our study, increasing BMI is also associated with decrease of WSI. High BMI can lead to a state of chronic inflammation through excretion of proinflammatory substances, which may reduce iron availability due to hepcidin-mediated reduced iron absorption. ${ }^{15} 49$ In conclusion, income effects iron alimentation by nutrition, stress and physical activity. Another predictor for iron-related alimentation is education. In our study, we could not show any significant correlation between the laboratory parameters and educational status. But previous studies describe lower educational status is associated with unhealthy nutrition among children through poorer nutritional knowledge on iron content, meaningful food combinations, portions of implemented food and eating practices. ${ }^{50} 51$ Additionally, education influences health knowledge and healthy behaviour, for example, awareness of the positive influence of sports and the negative effects of stress. ${ }^{50-52}$ However, only a small number of studies have examined iron-related alimentation in this context. The results of our study show that occupational status has no significant association to iron-related parameters. Children's behaviour is often learnt by imitating parents' behaviour. Therefore, the education of the parents might influence the eating behaviour of the child. These behavioural effects on child health have been previously described..$^{50} 53$

Other findings of our study are that the distribution of haemoglobin, ferritin and transferrin is associated with gender and pubertal stage. On the one hand, this can be due to the physiological differences in iron metabolism between boys and girls, ${ }^{35}$ for example, with the onset of menses. ${ }^{10}$ On the other hand, existing data show that girls seem to be more vulnerable to the influences of social inequalities in general. ${ }^{53}$

However, it is important to note that there are certain limitations to our study. First, the study population is unequally distributed with over-representation of families with medium SES and children with 6 to 12 years of age. Second, the group of children with low SES is under-represented. The share of LIFE Child families with a monthly net income of more than $€ 2000(69.4 \%)$ is twice as big as the respective share of households in the city of Leipzig $(34 \%) .^{55}$ Further, it is necessary to state the voluntary nature of patients' recruitment. Thus, the initiative of the participants and their interest in health are necessary prerequisites and probably less pronounced in the lower social stratum. ${ }^{56}$ Therefore, it is tempting plausible that the observed effects are even more pronounced in the actual population since our study cohort consists of an unintended selection of healthier and more health-conscious families. Furthermore, it is difficult to access the differences in distribution, because a small shift in the laboratory parameters does not necessarily mean a child or adolescent is in danger of being iron deficient because the normal ranges of laboratory values are widely spread, especially for ferritin and transferrin. This is due to a possible influence of inflammation, infections, malignancies and so on; what we cannot exclude completely although our study is based on healthy volunteers. It would have been useful to analyse other laboratory parameters important for clinical practice, such as transferrin saturation. At least, the cross-sectional study design provides very restricted information on causal connections. Further studies are needed to evaluate potential causes for the examined parameters.

In conclusion, our study is the first to show that the distribution of iron-related parameters and the appearance of anaemia is associated to SES, gender and pubertal stage. Furthermore, rising WSI correlates to higher values for physical activity and lower BMI. These results are based on the usage of z-scores, which allows comparable results across different age groups and presents physiological courses. These results might be a benefit in 
prevention and diagnostic process of iron deficiency and anaemia and support further studies to deepen the topic. Furthermore, there is high political potential concerning the influence on food pricing and access to free sporting activities. By promoting the results of our study in daily clinical routine, we hope for earlier detection of possible deficiencies and timely diagnostic measures to allow therapeutic actions.

\section{Author affiliations}

${ }^{1}$ Clinic and Polyclinic for Pediatrics and Adolescent Medicine, Center for Pediatric Research, University Hospital Leipzig (Public-Law Institution), Leipzig, Germany ${ }^{2}$ LIFE-Leipzig Research Center for Diseases of Civilization, University of Leipzig, Leipzig, Germany

${ }^{3}$ Institute for Medical Informatics, Statistics and Epidemiology, University of Leipzig, Leipzig, Germany

${ }^{4}$ Institute for Laboratory Medicine, Clinical Chemistry, and Molecular Diagnostics, University Hospital Leipzig (Public-Law Institution), Leipzig, Germany

${ }^{5}$ Institute for Sociology, University of Leipzig, Leipzig, Germany

${ }^{6}$ Institute of Medical Sociology, Martin Luther University of Halle-Wittenberg, Halle (Saale), Germany

Contributors The data analysis and production of the manuscript were done by $\mathrm{KR}$ in collaboration with MV and CE with respect to statistics; with UC and KH with respect to laboratory medicine; with $\mathrm{HL}$ and $\mathrm{MR}$ with respect to medical sociology; with UW and WK with respect to pediatrics. All authors are responsible for the entire contents of this article and have approved the submission of the manuscript.

Funding This work was supported by LIFE (Leipzig Research Center for Civilization Diseases). LIFE is funded by the European Union through the European Regional Development Fund (ERDF) and by the Free State of Saxony under the State Excellence Initiative. This is an interdisciplinary project. We acknowledge support from the German Research Foundation (DFG) andLeipzig University within the program of Open Access Publishing acknowledge support from the German Research Foundation (DFG) andLeipzig University within the program of Open Access Publishing.

\section{Competing interests None declared.}

Patient consent Detail has been removed from this case description/these case descriptions to ensure anonymity. The editors and reviewers have seen the detailed information available and are satisfied that the information backs up the case the authors are making.

Ethics approval Ethical Committee at the Medical Faculty, Leipzig University (Reg. no 264-10-19042010).

Provenance and peer review Not commissioned; externally peer reviewed.

Data sharing statement The statistical code and dataset are available from the corresponding author. A list of the approved assessments of the LIFE Child Study, which partly contains additional unpublished data, is inserted in the supplementary files.

Open access This is an open access article distributed in accordance with the Creative Commons Attribution Non Commercial (CC BY-NC 4.0) license, which permits others to distribute, remix, adapt, build upon this work non-commercially, and license their derivative works on different terms, provided the original work is properly cited and the use is non-commercial. See: http://creativecommons.org/ licenses/by-nc/4.0/

(c) Article author(s) (or their employer(s) unless otherwise stated in the text of the article) 2018. All rights reserved. No commercial use is permitted unless otherwise expressly granted.

\section{REFERENCES}

1. Richter M, Hurrelmann K. Health inequalities (Gesundheitliche Ungleichheit). Wiesbaden: VS Verlag für Sozialwissenschaften, 2009.

2. Mokdad AH, Forouzanfar MH, Daoud F, et al. Global burden of diseases, injuries, and risk factors for young people's health during 1990-2013: a systematic analysis for the Global Burden of Disease Study 2013. Lancet 2016;387:2383-401.
3. Chen E, Martin AD, Matthews KA. Socioeconomic status and health: do gradients differ within childhood and adolescence? Soc Sci Med 2006;62:2161-70.

4. Goldman D, Smith JP. The increasing value of education to health. Soc Sci Med 2011;72:1728-37.

5. Choi HJ, Lee HJ, Jang HB, et al. Effects of maternal education on diet, anemia, and iron deficiency in Korean school-aged children. BMC Public Health 2011;11:870.

6. Zimmermann MB, Hurrell RF. Nutritional iron deficiency. Lancet 2007;370:511-20.

7. Corsi DJ, Chow CK, Lear SA, et al. Shared environments: a multilevel analysis of community context and child nutritional status in Bangladesh. Public Health Nutr 2011;14:951-9.

8. Michaelsen KF. Feeding and nutrition of infants and young children: guidlines for the WHO European Region, with emphasis on the former Soviet countries. Copenhagen, Denmark: WHO Regional Office for Europe, 2000.

9. Ahman E, Allen H, Beaton G, et al. Iron deficiency update. 4th Report -the World Nutrition situation: nutrition throughout the life cycle. Geneva: United Nations System Standing Comittee on Nutrition, World Health Organization, 2000:36-41.

10. Beard JL. Iron requirements in adolescent females. J Nutr 2000;130(2S Suppl):440S-2.

11. Geyer S. Manifestation, explanation and reduction of health inqualities (Manifestation, Erklärung und Reduzierung gesundheitlicher Ungleichheiten). Ethik Med 2010;22:249-62.

12. Patton GC, Coffey C, Cappa C, et al. Health of the world's adolescents: a synthesis of internationally comparable data. Lancet 2012;379:1665-75.

13. Silva LS, Giuglian ER, Aerts DR. Prevalence and risk factors for anemia among children in Brazil (Prevalência e determinantes de anemia em crianças de Porto Alegre, RS, Brasil). Rev Saude Publica 2001;35:66-73

14. Mikolajczyk RT, Richter M. Associations of behavioural, psychosocial and socioeconomic factors with over- and underweight among German adolescents. Int $J$ Public Health 2008:53:214-20.

15. Cepeda-Lopez AC, Aeberli I, Zimmermann MB. Does obesity increase risk for iron deficiency? A review of the literature and the potential mechanisms. Int J Vitam Nutr Res 2010;80(4-5):263-70.

16. Reinhold $S$, Jürges H. Parental income and child health in Germany. Health Econ 2012;21:562-79.

17. DeLoughery TG. Microcytic anemia. N Engl J Med 2014:371:1324-31

18. Quante M, Hesse M, Döhnert M, et al. The LIFE child study: a life course approach to disease and health. BMC Public Health 2012;12:1021-34.

19. Association of the Scientific Medical Societies. Extended version of the guideline "iron deficiency anemia". $2010 \mathrm{http} / / / \mathrm{www} . \mathrm{awmf}$. org/ leitlinien/detail/l//025-021.html (accessed 31 Aug 2015).

20. World Health Organization, Centers for Disease Control and Prevention. Assessing the iron status of populations. 2nd edn. Geneva: World Health Organization, Dept. of Nutrition for Health and Development; Centers for Disease Control and Prevention, Division of Nutrition and Physical Activity, International Micronutrient Malnutrition Prevention and Control Program, 2007.

21. Davies M, Morgan A, Ziglio E. International health and development: investing in assets of individuals, communities and organizations. New York, London: Springer, 2010.

22. Ruhe AK, Wager J, Hirschfeld G, et al. Household income determines access to specialized pediatric chronic pain treatment in Germany. BMC Health Serv Res 2016;16:140.

23. Winkler J, Stolzenberg H. Adjusting the social status index for implementation in the health survey for chidren and adolescence (KiGGS) 2003/2006 (Adjustierung des Sozialen-Schicht-Index für die Anwendung im Kinder- und Jugendgesundheitssurvey (KiGGS) 2003/2006). Wismar: Hochsch., Fakultät für Wirtschaftswiss; HWSHochsch.-Wismar-Service, 2009.

24. Marshall WA, Tanner JM. Variations in pattern of pubertal changes in girls. Arch Dis Child 1969;44:291-303.

25. Marshall WA, Tanner JM. Variations in the pattern of pubertal changes in boys. Arch Dis Child 1970;45:13-23.

26. Worth A, Woll A, Albrecht C, et al. MoMo-Längsschnittstudie "Physical fitness and physical activity as determinants of health development in children and adolescents": Testmanual zu den motorischen Tests und den anthropometrischen Messungen (KIT Scientific Reports; 7700): KIT Scientific Publishing, 2015.

27. Woll A, Kurth BM, Opper E, et al. The 'Motorik-Modul' (MoMo): physical fitness and physical activity in German children and adolescents. Eur J Pediatr 2011:170:1129-42. 
28. World Health Organization. Physical status: the use and interpretation of anthropometry. Report of a WHO Expert Committee. Geneva: World Health Organization, 1995.

29. Cole TJ, Green PJ. Smoothing reference centile curves: the LMS method and penalized likelihood. Stat Med 1992;11:1305-19.

30. Cole TJ, Stanojevic S, Stocks J, et al. Age- and size-related reference ranges: a case study of spirometry through childhood and adulthood. Stat Med 2009;28:880-98

31. R Core Team. R: A Language and Environment for Statistical Computing. 2014 http://www.R-project.org/ (accessed 15 July 2016).

32. Rigby RA, Stasinopoulos DM. Generalized additive models for location, scale and shape (with discussion). J R Stat Soc Ser C Appl Stat 2005;54:507-54.

33. Stasinopoulos DM, Rigby RA. Generalized additive models for location, scale and shape (GAMLSS) for modeling reference. J Stat Softw 2007;23:1-46.

34. World Health Organization. WHO Global Database on Child Growth and Malnutrition. Geneva: World Health Organization, 1997.

35. Rieger K, Vogel M, Engel C, et al. Reference intervals for iron-related blood parameters: results from a population-based cohort study (LIFE Child). LaboratoriumsMedizin 2016;40.

36. Vogel M, Kirsten T, Kratzsch J, et al. A combined approach to generate laboratory reference intervals using unbalanced longitudinal data. J Pediatr Endocrinol Metab 2017;30:767-73.

37. Case A, Lubotsky D, Paxson C. Economic status and health in childhood: the origins of the gradient. Am Econ Rev 2002;92:1308-34.

38. Khanam R, Nghiem HS, Connelly LB. Child health and the income gradient: evidence from Australia. J Health Econ 2009;28:805-17.

39. Io M, Council NR, Caswell JA, et al. Supplemental nutrition assistance program: examining the evidence to define benefit adequacy: National Academies Press, 2013.

40. Darmon N, Drewnowski A. Contribution of food prices and diet cost to socioeconomic disparities in diet quality and health: a systematic review and analysis. Nutr Rev 2015;73:643-60.

41. Rydén PJ, Hagfors L, cost D. Diet cost, diet quality and socioeconomic position: how are they related and what contributes to differences in diet costs? Public Health Nutr 2011;14:1680-92.

42. Laraia BA, Leak TM, Tester JM, et al. Biobehavioral factors that shape nutrition in low-income populations: a narrative review. Am J Prev Med 2017;52(2S2):S118-26.

43. Bhattacharya J, Currie J, Haider S, Poverty HS. Poverty, food insecurity, and nutritional outcomes in children and adults. J Health Econ 2004;23:839-62.
44. Hemmingsson E. A new model of the role of psychological and emotional distress in promoting obesity: conceptual review with implications for treatment and prevention. Obes Rev 2014;15:769-79

45. Moore CJ, Cunningham SA, position S. Social position, psychological stress, and obesity: a systematic review. J Acad Nutr Diet 2012;112:518-26.

46. Devine CM, Jastran $M$, Jabs J, et al. "A lot of sacrifices:" workfamily spillover and the food choice coping strategies of low-wage employed parents. Soc Sci Med 2006;63:2591-603.

47. Nairz M, Theurl I, Swirski FK, et al. "Pumping iron"-how macrophages handle iron at the systemic, microenvironmental, and cellular levels. Pflugers Arch 2017;469:397-418.

48. Truthmann J, Mensink GB, Richter A. Relative validation of the KiGGS Food Frequency Questionnaire among adolescents in Germany. Nutr J 2011;10:133.

49. Hardy LL, Mihrshahi S, Gale J, et al. 30-year trends in overweight, obesity and waist-to-height ratio by socioeconomic status in Australian children, 1985 to 2015. Int J Obes 2017;41:76-82.

50. Neumark-Sztainer D, Hannan PJ, Story M, et al. Family meal patterns: associations with sociodemographic characteristics and improved dietary intake among adolescents. J Am Diet Assoc 2003;103:317-22.

51. Ahsan KZ, Arifeen SE, Al-Mamun MA, et al. Effects of individual, household and community characteristics on child nutritional status in the slums of urban Bangladesh. Arch Public Health 2017;75:9.

52. Dorsey JL, Manohar S, Neupane S, et al. Individual, household, and community level risk factors of stunting in children younger than 5 years: Findings from a national surveillance system in Nepal. Matern Child Nutr 2018;14.

53. Richter M, Körholz D. The social dimension of adolescent health: challenges for paediatric research and practice. Klin Padiatr 2015;227:193-8.

54. Richter $M$, Erhart $M$, Vereecken $C A$, et al. The role of behavioural factors in explaining socio-economic differences in adolescent health: a multilevel study in 33 countries. Soc Sci Med 2009;69:396-403.

55. City of Leipzig, Office for Statistics and Elections. Statistical yearbook 2016, 2016. http://statistik.leipzig.de/statpubl/content/12 statistik-und-wahlen/jahrbuecher/Kapitel02.pdf (accessed 09 Feb 2016)

56. Filc D, Davidovich N, Novack L, et al. Is socioeconomic status associated with utilization of health care services in a single-payer universal health care system? Int J Equity Health 2014;13:115. 